\title{
Misguided drug advice for COVID-19
}

Cite as: G. A. FitzGerald, Science

10.1126/science.abb8034 (2020).

\section{Garret A. FitzGerald}

Institute for Translational Medicine and Therapeutics, Perelman School of Medicine, University of Pennsylvania, Philadelphia, PA 19104-5158, USA.

Email: garret@upenn.edu

As pandemic coronavirus disease 2019 (COVID-19) continues to accelerate, French Health Minister, Olivier Véran has confused matters by claiming on Twitter that antiinflammatory drugs like ibuprofen or cortisone could aggravate the infection (1). However, scientific evidence does not indicate that nonsteroidal anti-inflammatory drug (NSAID) consumption puts patients who otherwise might have mild or asymptomatic infection by severe acute respiratory syndrome-coronavirus 2 (SARS-CoV-2)-the virus that causes COVID-19-at risk of more severe disease. People taking NSAIDs for other reasons should not stop doing so for fear of increasing their COVID-19 risk.

NSAIDS work by suppressing prostaglandin synthases 1 and 2, colloquially known as COX-1 and COX-2. These enzymes produce prostaglandins (PGs), lipids that can trigger pain and fever. COX-2 produces most of the PGs relevant to pain and inflammation. NSAIDs selective for inhibiting COX2 include celecoxib and diclofenac; ibuprofen is an NSAID that blocks both COXs. Minister Véran advised people to take paracetamol (acetaminophen) instead to treat a fever (I), but this guidance only adds to the confusion given that acetaminophen is also an NSAID (2).

We don't know with certainty whether NSAIDS could lead to more severe COVID-19 symptoms because PGs, such as PGE2, PGD2, and prostacyclin (PGI2) can both promote and restrain inflammation. For example, the infection of certain immune cells (microglia) with a related coronavirus (not the one that causes COVID-19) activates a proinflammatory response (the inflammasome) to combat the pathogen; however, PGD2 increases the expression of PYDC3, a putative inflammasome inhibitor, in certain immune cells in mice (3). The SARS coronavirus responsible for the 2003 outbreak directly binds to the COX-2 promotor and increases its expression (4), boosting PG production capacity, and there is also evidence that PGE2 inhibits SARS coronavirus replication (5). Indomethacin, an NSAID, blocks coronavirus RNA synthesis, but independently of COX inhibition (6). By contrast, COX2-dependent PGE2 attenuates the chronic antiviral lymphocyte response of unresolved viral infection (7). Based on these findings, if we see a clinical signal, we can rationalize it. But therein lies the challenge. Many clinical anecdotes remain stalled in biological plausibility.

The prospect of a rapid increase in COVID-19 cases prompts us to seek covariates of disease severity, from the consumption of certain drugs before infection, to human genetic variants (8), to demographic factors such as sex and environmental exposures. In the case of NSAIDs, commonly acquired without prescription, such determination requires documentation of drug exposure and evidence of PG suppression.

Considering all of this, should patients with clinically complicated SARS-CoV-2 infections be administered NSAIDs as a treatment? No. There is no evidence of benefit. If such a patient were also to have poor kidney function, maintenance of renal blood flow becomes critically dependent on vasodilator PGs, such as PGE2 and PGI2 (9). Such a situation might also predispose the patient to the gastrointestinal and cardiovascular complications of NSAIDs. However, until we have robust evidence, patients in chronic pain should continue to take their NSAIDs rather than turn to opiates. Given that the elderly appear to comprise the predominant at-risk group for severe COVID-19 at this time, an association between NSAIDs and the disease may merely reflect reverse causality. That is, infection makes you more susceptible to adverse effects of NSAIDs on the infection.

A similar rationale should be applied to evidence that coronaviruses use the angiotensin converting enzyme (ACE) 2 as a receptor for cellular entry (10). There has been speculation, but no clinical evidence, that consumption of ACE inhibitors might worsen the consequences of infection (11). Patients on ACE inhibitors should continue to take them rather than risk complications, such as stroke.

\section{REFERENCES AND NOTES}

1. K. Willsher, "Anti-inflammatories may aggravate Covid-19, France advises," The Guardian (2020).

2. F. Catella-Lawsonet al., N. Engl. J. Med. 345, 1809 (2001).

3. R. Vijay et al., Proc. Natl. Acad. Sci. U.S.A. 114, E5444 (2017).

4. W. Yan et al., Int. J. Biochem. Cell Biol. 38, 1417 (2006).

5. C. Amiciet al., Antivir. Ther. 11, 1021 (2006).

6. W. J. Sander et al., Front. Physiol. 8, 89 (2017).

7. K. Schaeuble et al., PLOS Biol. 17, e3000072 (2019).

8. Y. Cao et al., Cell Discov. 6, 11(2020).

9. T. Grosser, S. Fries, G. A. FitzGerald, J. Clin. Invest. 116, 4 (2006)

10. K. Wu, W. Li, G. Peng, F. Li, Proc. Natl. Acad. Sci. U.S.A. 106, 19970 (2009).

11. L. Fang, G. Karakiulakis, M. Roth, Lancet Resp. Med. 10.1016/S22132600(20)30116-8 (2020).

\section{COMPETING INTERESTS}

G.A.F. has NIH support (R01HL141912-01) for atherosclerosis, prostaglandin inhibition, and checkpoint blockade. He is on the board of Kings Health Partners, London, UK; a member of the Governing Authority of the University of Limerick, Ireland; and on the Scientific Advisory Board of the Italian Science Agency. He is a Chief Scientific Advisor for Science Translational Medicine.

Published online 20 March 2020

10.1126/science.abb8034 